\title{
Endothelial Function and Inflammatory Activity in Patients with Recent Onset of Severe Plaque Psoriasis
}

\author{
Lotus Mallbris, ${ }^{*}$, John Pernow ${ }^{\mathrm{b}}$ and Mona Ståhle ${ }^{\mathrm{a}}$
}

\author{
Units of ${ }^{a}$ Dermatology and ${ }^{b}$ Cardiology, Department of Medicine, Karolinska University Hospital, Solna, Karolinska \\ Institutet, Stockholm, Sweden
}

\begin{abstract}
Background: Psoriasis is associated with premature atherosclerosis and cardiovascular co-morbidity and mortality.

Objective: To determine endothelial function in severe psoriasis at onset compared to that of matched healthy controls

Methods: Using high-resolution ultrasound, we measured the diameter of brachial artery at rest, during reactive hyperemia, and after sublingual nitroglycerin. We compared twenty adult individuals at psoriasis onset to matched healthy controls. Demographic characteristics, lipid profile and high-sensitive C-reactive protein were assessed.

Results: There were no significant differences in endothelium-dependent and -independent dilatation between psoriasis patients and control individuals. Patients with psoriasis had significantly higher concentration of high-sensitive Creactive-protein, reflecting the systemic inflammatory response.

Conclusion: This is the first pilot study evaluating endothelial function in individuals with early plaque psoriasis. The study shows that patients with severe psoriasis at onset do not exhibit endothelial dysfunction compared to control individuals.
\end{abstract}

Keywords: Atherosclerosis, cardiovascular disease, disease onset, endothelial function, psoriasis.

\section{INTRODUCTION}

Psoriasis (Ps) is a common, complex and chronic skin disorder affecting $2-3 \%$ of the adults in the West. Despite the substantial immunogenetic knowledge that has been amassed, the etiology of Ps is only partially understood. However, accumulating evidence indicate that Ps should be considered as a systemic inflammatory condition analogous to other inflammatory immune disorders, such as Rheumatoid arthritis (RA) and systemic lupus erythematosus (SLE) [1]. In support of the systemic nature of the inflammatory process in Ps, several studies have demonstrated an increased level of C-reactive protein (CRP), a hallmark of systemic inflammation, in patients with Ps [2-4]. Similar to RA and SLE, overwhelming data indicate that individuals with Ps, especially those with severe disease, have an excessive risk of cardiovascular disease (CVD) co-morbidity and mortality $[1,5-8]$. There is more than three-fold increased relative risk of myocardial infarction (MI) and mortality from MI in young individuals ( $<40 \mathrm{yrs}$ ) with severe Ps compared to controls $[6,7]$. Interestingly, the excessive relative risk of MI seems to persist even after adjustment for the major risk factors for CVD, suggesting that psoriasis might be considered as an independent risk factor for MI [7].

Furthermore, a recent spiral computer tomography study demonstrated a significantly higher prevalence and severity of coronary artery calcification as an indicator for CVD in

*Address correspondence to this author at the Department of Medicine, Dermatology Unit, Karolinska Institutet, Karolinska University Hospital, Solna, SE-171 76, Stockholm, Sweden; Tel: +46-7-336 698 59; Fax: +46-8517778 51; E-mail: lotus.mallbris@ki.se patients with Ps compared to control subjects without Ps, supporting the notion that Ps is an independent risk factor for CVD [1]. The underlying pathological mechanisms for such an association are unclear. However, inflammation is now recognized as being pivotal in the pathogenesis of atherosclerosis $[9,10]$ and current data strongly suggest that chronic inflammation in Ps may play a key role in many, if not all, stages of developing CVD [11, 12]. To date, it has not been studied whether the intensity of inflammation, measured as Ps severity, or the duration of chronic inflammation are more important in enhancing atherosclerosis and CVD co-morbidity in individuals with severe Ps.

Endothelial dysfunction is a barometer for the early stage of atherosclerosis [13] and a predictor for cardiovascular events $[14,15]$. Both RA and SLE are associated with endothelial dysfunction [16-21]. Flow-mediated vasodilatation (FMD) of the brachial artery by using a high resolution ultrasound is a non-invasive and extensively validated method for assessing systemic endothelial function. It is unclear whether the inflammation observed in Ps also is associated with endothelial dysfunction. The aim of the present pilot study was to investigate if endothelium-dependent vasodilatation, quantified by FMD, is impaired in adult individuals at the onset of severe Ps $(<12 \mathrm{mo})$ and without prior CVD, in comparison with healthy matched controls.

\section{MATERIALS AND METHODOLOGY}

\section{Subjects and Study Design}

Cases were selected from the Stockholm Psoriasis Cohort (SPC) which is a prospective cohort consisting of adult individuals with newly onset $(<12 \mathrm{mo})$ of Ps followed at the 
Dermatology Clinic at Karolinska University Hospital, Solna, Stockholm, Sweden. The recruitment process has been described [22]. In the present study, a total of twenty adult individuals between 16 to 40 years old, with severe plaque psoriasis were enrolled. All patients were examined by one dermatologist (L.M.) at Department of Dermatology at Karolinska University Hospital Solna, Stockholm, Sweden. Measurement of skin disease severity was performed once at the enrollment in the SPC and again at the enrollment in the present study, using the Psoriasis Area and Severity Index (PASI) [23]. Only individuals with plaque phenotype and a PASI-score above 12 , representing severe psoriasis [24], and with no pervious history of CVD were included. The study coordinator randomly selected twenty patients from SPC based on the study criteria for participation. A population based healthy control individual was selected as a stratified random sample, taking age and sex into consideration. Participation involved attending a clinical examination including an ultrasound examination for evaluation of FMD (see below), providing blood samples, and completing a questionnaire regarding smoking and snuffing habits, ongoing medication, and for female subjects providing the date of the first day of their last menstruation. Body mass index (BMI) was calculated for all subjects using the current weight $(\mathrm{kg})$ at the date of examination. The study was approved by the Ethical Committee of the Karolinska University Hospital, and all subjects gave their informed consent. The study was performed according to the Declaration of Helsinki Principles.

\section{Laboratory Examinations}

Blood samples for assessment of blood lipids and highsensitive C-reactive protein (hs-CRP) were collected after an overnight fast. Fasting plasma concentrations of total cholesterol and cholesterol (C) in plasma lipoproteins (very lowdensity lipoproteins (VLDL), low-density lipoproteins (LDL) and high-density lipoproteins (HDL)), and total concentration of triglycerides (TG) were determined by a combination of preparative ultracentrifugation, precipitation of ApoB-containing lipoproteins, and lipid analyses [25]. Measurements of apolipoprotein A-1 (Apo A) and apolipoprotein B (Apo B) were performed by using kits made by SYNCHRON LX 20 systems (Beckman, Inc. Fullerton, Calif). Serum Lipoprotein (a) was determined by the IMMAGE Immunochemistry System, (Beckman, Inc), and hsCRP was measured by the Dade-Behring method (N High Sensitivity CRP System; Dade Behring Co Inc, Newark, Del).

\section{Measurement of Flow- and Nitroglycerin Mediated Dila- tation}

FMD and nitroglycerin-mediated vasodilatation (NMD) for the determination of endothelium-dependent and independent dilatation, respectively were assessed at the Clinical Cardiovascular Research Laboratory at the Karolinska University Hospital, Solna, Stockholm, Sweden, by an experienced investigator according to Celermajer et al. [26]. The investigations were performed in a quiet, dimly lit room with the subjects in the supine position. All study subjects were fasting and were instructed to refrain from alcohol, caffeine, nicotine, antioxidants and vasoactive drugs during the past $24 \mathrm{~h}$. Subjects lay supine for $20 \mathrm{~min}$ before the FMD was conducted. In brief, the measurements were made noninvasively using a high-resolution ultrasound scanner (Acuson $128 \mathrm{XP} / 10$ c; Siemens, Mountain View, California, USA) with a 7-MHz linear array transducer, as described [27]. To minimize variability we used a special clamp to keep the probe in the same position during the investigation. Baseline images were saved every third second during one min and a mean value was calculated from these values. Subsequently a blood pressure cuff, positioned below the elbow, was inflated to $260 \mathrm{mmHg}$ for five min. The artery was continuously imaged for three min during the hyperemia following release of the cuff pressure to determine FMD. A mean value was calculated from five recordings at maximum dilatation. Endothelium-independent vasodilatation was determined following sublingual administration of nitroglycerin (0.4 $\mathrm{mg}$ ). All images were analyzed using proprietary software (Brachial analyzer $^{\mathbb{B}}$, Medical Imaging Applications, Iowa City, IA, USA) by a technician, blinded to the case-control status. The maximum lumen diameter, found through beatto-beat analysis, was measured using an automated contour detection system. Lumen diameter was defined as the distance between the intima of the far and near vessel walls. Percent dilatation was calculated as maximal lumen diameter after ischemia or nitroglycerine minus lumen diameter at baseline divided by lumen diameter at baseline. The coefficient of variation for FMD determination on two study occasions was $18 \%$. Systolic (SBP) and diastolic (DBP) blood pressure and heart rate were determined at rest, and following the FMD.

\section{Statistical Analysis}

Results are presented as mean, median and standard deviation (SD). Group comparisons were performed using the Student's t-test. A $P$ value less than 0.05 was considered to be statistically significant. Statistica 6.1 (StatSoft Inc., Tulsa, Oklahoma, USA) was used for all statistical analyses. The correlation between continuous variables was assessed using Pearson's linear correlation coefficients (r).

\section{RESULTS}

The characteristics of patients and control individuals are shown in Table 1. In brief, demographic data were similar for patients and control individuals except for nicotine usage. Patients with psoriasis slightly more often were current daily cigarette smokers than control subjects (Table 1). All subjects were young adults $(<40 \mathrm{yrs})$, with a median age of 29.5 years for both patients and control individuals (Table 1). The distribution of male $(n=9)$ and female $(n=11)$ was the same for patients and controls. No subject had diabetes mellitus, or ongoing basal reactive medications, psychiatric medication or medication that could affect the blood lipids (such as statins, or acitretin etc.). Patients had a resent onset of psoriasis with median four months of disease duration (mean $\pm \mathrm{SD}$ : $4.8 \pm 3.7$ ) and without any previous systemic treatment. Four individuals with psoriasis required inpatient care at disease onset as a consequence of severity of the disease.

There was no significant difference between patients and control subjects with regard to body mass index (BMI), systolic and diastolic blood pressure, blood lipid profile, including total plasma cholesterol and triglycerides, lipoprotein fractions of cholesterol (LDLc, VLDLc, HDLc), lipoprotein (a) and apolipoproteins. There was however a significant 
Table 1. Characteristics of Individuals with Severe Psoriasis at Onset $(n=20)$ and Matched Healthy Controls $(n=20)$

\begin{tabular}{|c|c|c|c|c|c|}
\hline & \multicolumn{2}{|c|}{ Severe Psoriasis at Onset } & \multicolumn{2}{|c|}{ Healthy Controls } & $p$ \\
\hline Age & 29.6 & 7.3 & 29.5 & 7.4 & ns \\
\hline BMI $\left(\mathrm{kg} / \mathrm{m}^{2}\right)$ & 26.3 & 6.2 & 24.2 & 3.5 & $\mathrm{~ns}$ \\
\hline \multicolumn{6}{|l|}{ Blood pressure (mmHg) } \\
\hline \multicolumn{6}{|l|}{ Blood lipids } \\
\hline Total cholesterol $(\mathrm{mmol} / \mathrm{L})$ & 4.8 & 0.9 & 5.2 & 1.0 & ns \\
\hline LDLc & 2.9 & 0.8 & 3.2 & 0.8 & ns \\
\hline VLDLc & 0.4 & 0.3 & 0.2 & 0.1 & ns \\
\hline \multicolumn{6}{|l|}{ Apolipoproteins (Apo)(g/L) } \\
\hline Apo A & 1.4 & 0.3 & 1.4 & 0.2 & ns \\
\hline Apo B & 0.9 & 0.3 & 0.8 & 0.2 & ns \\
\hline Apo B/Apo A & 0.7 & 0.3 & 0.6 & 0.2 & ns \\
\hline Lipoprotein (a) (g/L) & 0.2 & 0.2 & 0.3 & 0.3 & ns \\
\hline hs-CRP (mg/L) & 4.5 & 5.3 & 1.6 & 1.9 & $<0.05$ \\
\hline Disease duration (months) & 4.8 & 3.7 & --- & --- & \\
\hline PASI & 14.3 & 4.8 & --- & --- & \\
\hline
\end{tabular}

difference in hs-CRP concentration between patients and control subjects (mean $\pm \mathrm{SD}: 4.5 \pm 5.3$ in psoriasis patients vs $1.6 \pm 1.9$ in control individuals; $p<0.05$ ) (Table 1 ).

We did not detect any differences in baseline brachial artery diameter, endothelium-dependent FMD or endothelium-independent dilatation between patients with severe Ps and control individuals (Table 2). Furthermore, there were no significant associations between Ps disease severity index or hs-CRP and FMD $(\mathrm{r}=0.1)$ in the present study.

\section{DISCUSSION}

Cardiovascular disease is emerging as an important cause of co-morbidity $[1,7]$ and mortality [6] in severe Ps. Psoriasis is a chronic inflammatory immune disorder and as such, it has been suggested as a possible independent risk factor for development of coronary artery calcification [1] and myocardial infarction [7]. Consistent with this notion, other chronic inflammatory immune disorders have been identified as independent risk factors for CVD. Recent studies have demonstrated that patients with psoriasis arthritis (PsA) [28], RA [16-18] and SLE [19-21], have impaired endothelial function reflecting an early stage of atherosclerosis [29, 30]. The endothelial dysfunction seems to appear prior to any overt CVD and in the absence of classic cardiovascular risk factors, indicating that systemic inflammatory, a key feature in PsA, RA and SLE, may play a pivotal role in the increased prevalence of cardiovascular complications in these patients.
In the present study, we assessed FMD, a marker of endothelial function and an indicator for the earliest stage of atherosclerosis [29, 30]. We compared patients with severe Ps within $<12$ months of disease onset to age- and sexmatched healthy control individuals. We also measured nitroglycerin-mediated vasodilatation, representing endothelial independent dilatation. Our results show that Ps patients with severe disease at onset, without clinically evident CVD do not exhibit endothelial dysfunction.

We used PASI to evaluate disease severity in patients with Ps. Despite many limitations, PASI is to date the most commonly used instrument to evaluate Ps severity in clinical practice and randomized controlled trials [24]. In accordance with previous studies, we considered PASI score greater than 12 as severe [24], and as an indicator of intensity of the systemic inflammatory process in patients with Ps. Furthermore, we used hs-CRP to confirm the magnitude of the inflammation in patients. Indeed, the concentration of hs-CRP was significantly higher in patients with Ps compared to that of healthy individuals. Epidemiologic studies suggest that an increase in hs-CRP concentration may be an independently predictor for CVD, even after adjustment for other cardiovascular risk factors [31, 32]. Furthermore, accumulating evidence from experimental studies point to hs-CRP not only being a predictor of CVD but also playing an active role in the development and progression of atherosclerosis [33]. However, in the present study there was no correlation between elevated hs-CRP concentration in Ps patients and 
Table 2. Brachial Artery Flow Endothelium-Dependent (FMD) and Endothelium-Independent Nitroglycerin Induced Dilation (NMD) in Individuals with Severe Psoriasis at Onset and Normal Matched Healthy Controls

\begin{tabular}{|c|c|c|c|c|c|}
\hline & \multicolumn{2}{|c|}{ Severe Psoriasis at Onset } & \multicolumn{2}{|c|}{ Healthy Controls } & \multirow{2}{*}{$p$} \\
\hline & Mean & SD & Mean & SD & \\
\hline \multicolumn{6}{|l|}{ FMD (dependent endothelial dilation) } \\
\hline $\mathrm{B} 1(\mathrm{~mm})$ & 3.3 & 0.6 & 3.4 & 0.6 & ns \\
\hline B2 (mm) & 3.6 & 0.6 & 3.7 & 0.6 & ns \\
\hline B2-B1 (mm) & 0.4 & 0.2 & 0.3 & 0.1 & ns \\
\hline$(\mathrm{B} 2-\mathrm{B} 1) / \mathrm{B} 1 \times 100(\%)$ & 11.0 & 5.3 & 10.4 & 5.2 & ns \\
\hline \multicolumn{6}{|c|}{ NMD (independent endothelial dilation) } \\
\hline $\mathrm{B} 1$ & 3.4 & 0.6 & 3.5 & 0.6 & ns \\
\hline B2 & 4.1 & 0.7 & 4.1 & 0.5 & ns \\
\hline B2-B1 (mm) & 0.7 & 0.2 & 0.5 & 0.4 & ns \\
\hline$(\mathrm{B} 2-\mathrm{B} 1) / \mathrm{B} 1 \times 100(\%)$ & 20.5 & 6.9 & 20.7 & 6.8 & ns \\
\hline
\end{tabular}

B1: diameter of brachial artery at rest, B2: diameter of brachial artery after inducing shearing stress (in FMD measurement) or after administration of sublingual nitroglycerin (in NMD measurement). B2-B1 represents absolute value of vessel dilation (flow- or nitroglycerin-mediated dilation), B2-B1/B1*100 represents vessel dilation as the percent of baseline value. ns: not significant.

FMD $(r=0.1)$. In spite of this finding, we hypothesize that while the early acute severe inflammatory phase of Ps seem to not be associated with endothelial dysfunction in Ps patients, the sustained and chronic refractory inflammation present in chronic plaque Ps might be important. To confirm such an association, further studies are needed, and a followup study of the patients investigated in the present study would be of value. To date several studies indicate an association of CVD and Ps [1, 5-8]. However, these studies have included only patients with long duration of active and more persistent inflammation. Ludwig et al. recently demonstrated a significantly increased prevalence and severity of coronary artery calcification in psoriatics [1]. However the mean disease duration of Ps in that study was 23 years [1]. Also, epidemiological studies that demonstrated higher risk of CVD in severe out- and inpatients with Ps, included patients with long disease duration [5-8]. Our study constitutes the first pilot attempt to specifically assess whether endothelial dysfunction, as an indicator of the earliest stage of atherosclerosis, is present in patients with severe plaque Ps, already at the initial phase of disease. To distinguish between the importance of severity of inflammation and disease duration as risk factor for CVD, further studies in patients with chronic plaque Ps, without prior CVD are needed. Hopefully such studies, in addition to the one presented here, will further our understanding of the pathomechanisms underlying CVD comorbidity and mortality in Ps and help prevent these conditions in Ps.

\section{ABBREVIATIONS}

BMI = Body mass index

$\mathrm{CI}=$ Confidence interval

CVD $=$ Cardiovascular disease

$\mathrm{DBP}=$ Diastolic blood pressure

FMD $=$ Flow mediated dilatation

HDLc = High-density lipoprotein cholesterol
hs-CRP = high-sensitive C-reactive protein

LDLc = Low-density lipoprotein cholesterol

MI = Myocardial infarction

$\mathrm{NMD}=$ Nitroglycerin-mediated vasodilatation

PASI = Psoriasis Area and Severity Index

Ps $=$ Psoriasis

PsA $=$ Psoriasis arthritis

RA $=$ Rheumatoid arthritis

SBP $=$ Systolic blood pressure

$\mathrm{SD}=$ Standard deviation

SLE $=$ Systemic lupus erythematosus

$\mathrm{TG}=$ Triglycerides

\section{ACKNOWLEDGEMENTS}

Supported by funds from Swedish Heart-Lung Foundation, Swedish Research Council (10857), Swedish Psoriasis Association, Swedish Society of Dermatology and Venereology, Welander-Finsen Foundation, Karolinska Institutet and Schering-Plough. Ann Lindström is gratefully acknowledged for skilful technical assistance.

\section{REFERENCES}

[1] Ludwig RJ, Herzog C, Rostock A, et al. Psoriasis: a possible risk factor for development of coronary artery calcification. Br J Dermatol 2007; 156: 271-6.

[2] Vanizor Kural B, Orem A, Cimsit G, Yandi YE and Calapoglu M. Evaluation of the atherogenic tendency of lipids and lipoprotein content and their relationships with oxidant-antioxidant system in patients with psoriasis. Clin Chim Acta 2003; 328: 71-82.

[3] Chodorowska G, Wojnowska D and Juszkiewicz-Borowiec M. Creactive protein and alpha2-macroglobulin plasma activity in medium-severe and severe psoriasis. J Eur Acad Dermatol Venereol 2004; 18: 180-3.

[4] Rocha-Pereira P, Santos-Silva A, Rebelo I, et al. The inflammatory response in mild and in severe psoriasis. Br J Dermatol 2004; 150 917-28. 
[5] Poikolainen K, Karvonen J and Pukkala E. Excess mortality related to alcohol and smoking among hospital-treated patients with psoriasis. Arch Dermatol 1999; 135: 1490-3.

[6] Mallbris L, Akre O, Granath F, et al. Increased risk for cardiovascular mortality in psoriasis inpatients but not in outpatients. Eur J Epidemiol 2004; 19: 225-30.

[7] Gelfand JM, Neimann AL, Shin DB, et al. Risk of myocardial infarction in patients with psoriasis. JAMA 2006; 296: 1735-41.

[8] Shapiro J, Cohen AD, David M, et al. The association between psoriasis, diabetes mellitus, and atherosclerosis in Israel: a casecontrol study. J Am Acad Dermatol 2007; 56: 629-34.

[9] Ross R. Atherosclerosis--an inflammatory disease. N Engl J Med 1999; 340: 115-26.

[10] Hansson GK. Inflammation, atherosclerosis, and coronary artery disease. N Engl J Med 2005; 352: 1685-95.

[11] Tracy RP. Emerging relationships of inflammation, cardiovascular disease and chronic diseases of aging. Int J Obes Relat Metab Disord 2003; 27 Suppl 3: S29-34.

[12] Willerson JT and Ridker PM. Inflammation as a cardiovascular risk factor. Circulation 2004; 109: II2-10

[13] Vita JA and Keaney JF, Jr. Endothelial function: a barometer for cardiovascular risk? Circulation 2002; 106: 640-2.

[14] Lerman A and Zeiher AM. Endothelial function: cardiac events. Circulation 2005; 111:363-8.

[15] Corrado E, Rizzo M, Coppola G, et al. Endothelial dysfunction and carotid lesions are strong predictors of clinical events in patients with early stages of atherosclerosis: a 24-month follow-up study. Coron Artery Dis 2008; 19: 139-144.

[16] Szekanecz Z, Kerekes G, Der H, et al. Accelerated atherosclerosis in rheumatoid arthritis. Ann NY Acad Sci 2007; 1108: 349-58.

[17] Herbrig K, Haensel S, Oelschlaegel U, et al. Endothelial dysfunction in patients with rheumatoid arthritis is associated with a reduced number and impaired function of endothelial progenitor cells. Ann Rheum Dis 2006; 65: 157-63.

[18] Vaudo G, Marchesi S, Gerli R, et al. Endothelial dysfunction in young patients with rheumatoid arthritis and low disease activity. Ann Rheum Dis 2004; 63: 31-5.

[19] El-Magadmi M, Bodill H, Ahmad Y, et al. Systemic lupus erythematosus: an independent risk factor for endothelial dysfunction in women. Circulation 2004; 110: 399-404.

[20] Ahmad Y, Shelmerdine J, Bodill H, et al. Subclinical atherosclerosis in systemic lupus erythematosus (SLE): the relative contribution of classic risk factors and the lupus phenotype. Rheumatology (Oxford) $2007 ; 46: 983-8$
[21] Kiss E, Soltesz P, Der H, et al. Reduced flow-mediated vasodilation as a marker for cardiovascular complications in lupus patients. J Autoimmun 2006; 27: 211-7.

[22] Mallbris L, Larsson P, Bergqvist S, et al. Psoriasis phenotype at disease onset: clinical characterization of 400 adult cases. J Invest Dermatol 2005; 124: 499-504

[23] Fredriksson T and Pettersson U. Severe psoriasis--oral therapy with a new retinoid. Dermatologica 1978; 157: 238-44.

[24] Schmitt J and Wozel G. The psoriasis area and severity index is the adequate criterion to define severity in chronic plaque-type psoriasis. Dermatology 2005; 210: 194-9.

[25] Carlson K. Lipoprotein fractionation. J Clin Pathol Suppl (Assoc Clin Pathol) 1973; 5: 32-7.

[26] Celermajer DS, Sorensen KE, Gooch VM, et al. Non-invasive detection of endothelial dysfunction in children and adults at risk of atherosclerosis. Lancet 1992; 340: 1111-5.

[27] Lundman P, Eriksson MJ, Stuhlinger M, et al. Mild-to-moderate hypertriglyceridemia in young men is associated with endothelial dysfunction and increased plasma concentrations of asymmetric dimethylarginine. J Am Coll Cardiol 2001; 38: 111-6.

[28] Gonzalez-Juanatey C, Llorca J, Miranda-Filloy JA, et al. Endothelial dysfunction in psoriatic arthritis patients without clinically evident cardiovascular disease or classic atherosclerosis risk factors. Arthritis Rheum 2007; 57: 287-93

[29] Deanfield J, Donald A, Ferri C, et al. Endothelial function and dysfunction. Part I: Methodological issues for assessment in the different vascular beds: a statement by the Working Group on Endothelin and Endothelial Factors of the European Society of Hypertension. J Hypertens 2005; 23: 7-17.

[30] Brunner H, Cockcroft JR, Deanfield J, et al. Endothelial function and dysfunction. Part II: Association with cardiovascular risk factors and diseases. A statement by the Working Group on Endothelins and Endothelial Factors of the European Society of Hypertension. J Hypertens 2005; 23: 233-46.

[31] Rifai N and Ridker PM. High-sensitivity C-reactive protein: a novel and promising marker of coronary heart disease. Clin Chem 2001; 47: 403-11.

[32] Mitra B and Panja M. High sensitive C-reactive protein: a novel biochemical markers and its role in coronary artery disease. J Assoc Physicians India 2005; 53: 25-32.

[33] Libby P and Ridker PM. Inflammation and atherosclerosis: role of C-reactive protein in risk assessment. Am J Med 2004; 116 Suppl 6A: $9 \mathrm{~S}-16 \mathrm{~S}$ 